\title{
Comment on: 'KRAS and BRAF mutations are prognostic biomarkers in patients undergoing lung metastasectomy of colorectal cancer.' Variation in survival associated with proto-oncongenes is not evidence for effectiveness of lung metastasectomy
}

\author{
Giuseppe Cardillo ${ }^{1}$, Sahar Mokhles ${ }^{2}$, Norman Williams ${ }^{3}$, Fergus Macbeth ${ }^{4}$, Christopher Russell ${ }^{5}$ and Tom Treasure ${ }^{\star}, 6$
}

${ }^{1}$ Carlo Forlanini Hospital, Azienda Ospedaliera San Camillo Forlanini, Rome, Italy; ${ }^{2}$ Erasmus University Medical Center, Postbus 20403000 CA, Rotterdam, The Netherlands; ${ }^{3}$ Clinical Trials Group, Division of Surgery \& Interventional Science, Faculty of Medical Sciences, University College London, Room 4.24, 132 Hampstead Road, London NW1 2BX, UK; ${ }^{4}$ Wales Cancer Trials Unit, 6th Floor, Neuadd Meirionnydd, University Hospital of Wales, Heath Park, Cardiff CF14 4YS, UK; ${ }^{5}$ Division of Surgery \& Interventional Science, Faculty of Medical Sciences, University College London, Room 4.24, 132 Hampstead Road, London NW1 2BX, UK and ${ }^{6}$ Clinical Operational Research Unit, University College London, 4 Taviton Street, London WC1H OBT, UK

Sir,

The paper by Renaud et al (2015) begins and ends with acknowledgements that there has been no proof of effectiveness of pulmonary metastasectomy in colorectal cancer. The authors are correct. No difference in survival attributable to surgical removal of lung metastases has been shown in a controlled trial and yet it is regarded by them as 'steadily gaining acceptance'. Given the heterogeneity of patients with colorectal cancer, the variability in biology, the variety of treatments involved, and the variable time course between metastasectomy and death, a simple mechanistic cause and effect relationship cannot be derived from observational follow-up data (Glasziou et al, 2007; Fiorentino and Treasure, 2013a,b). Because of the lack of evidence for effectiveness the PulMiCC trial (Pulmonary Metastasectomy in Colorectal Cancer) seeks to answer that question (http://public.ukcrn.org.uk/search/ StudyDetail.aspx?StudyID=9018).

The differences in survival according to whether the patients had V-Ki-ras2 Kirsten rat sarcoma viral oncogene homologue (KRAS) or V-raf Murine sarcoma viral oncogene homologue $\mathrm{B} 1$ (BRAF) are striking as displayed in a Kaplan-Meier plot $(P<0.0001)$. Survival of patients with KRAS is worse than those with wild type and for the 19 patients with BRAF is lower still. On the basis of these data it would seem to be foolhardy to offer metastasectomy to BRAF positive patients. That much seems clear. The study shows the influence of oncogenes on survival but these are likely to be general prognostic factors, not predictors of the effectiveness of lung metastasectomy itself (Simms et al, 2013).

These are highly selected patients collected over 14 years, barely more than one per month. They were selected because of their very limited disease: 'All of the patients were considered completely cured of their primary tumour at the time of thoracic metastasectomy, and all pulmonary metastases were metachronous' (Renaud et al, 2015). They were asymptomatic, fit for surgery and individually picked for operation after careful assessment including a judgement of their 'survivability' (Treasure et al, 2015) at the time of selection. They are out in the longer lived tail of the survival curve for patients with metastatic colon cancer (Utley et al, 2008) and a survival effect attributable to metastasectomy cannot be estimated from these data.

The statistical interpretation lacks critical insight. The authors performed an analysis for the well know factors predictive of early death after metastasectomy: more than one metastasis, any elevation of CEA, an interval shorter than 3 years since primary resection and involvement of mediastinal lymph nodes (Gonzalez et al, 2013). The failure of CEA and short intervals to show in this analysis is a simple example of a beta error. Adverse prognostic features have been systematically excluded by clinical selection so variation in prognosis attributable to them cannot be excluded (Utley and Treasure, 2008).

The choice of citations is also misleading. The publications cited for metastasectomy are follow-up studies (Treasure and Utley, 2007) from 1984 to 1996 , reporting surgical series that closed $>20$ years ago. None of four systematic reviews were cited (Pfannschmidt et al, 2007; Fiorentino et al, 2010; Pfannschmidt et al, 2010; Gonzalez et al, 2013). It is well known that 'citation distortions create unfounded authority' (Greenberg, 2009). The problem has been well exemplified in lung metastasectomy for colorectal cancer (Fiorentino et al, 2011).

The authors conclude by saying: 'Lung metastasectomy of CRC is steadily gaining acceptance in the field of thoracic surgery, although it remains a subject of debate because of the absence of recent comparisons between simple follow-up and surgery'. There have been no randomised trials of metastasectomy in any tumour type and its effect on survival is therefore conjectural. But recently reported RCTs of the effect of intensive follow-up, with the intention of improving survival by operating on recurrent disease, show no survival benefit (Primrose et al, 2014; Treasure et al, 2014). This is indirect evidence casting further doubt on effectiveness of metastasectomy in colorectal cancer. It is hoped that the Cancer Research UK funded PulMiCC trial will bring some evidence to this important question.

\section{CONFLICT OF INTEREST}

The authors declare no conflict of interest.

\section{REFERENCES}

Fiorentino F, Hunt I, Teoh K, Treasure T, Utley M (2010) Pulmonary metastasectomy in colorectal cancer: a systematic review and quantitative synthesis. J R Soc Med 103: 60-66.

Fiorentino F, Treasure T (2013a) Pulmonary metastasectomy: are observational studies sufficient evidence for effectiveness? Ann Thorac Surg 96: 1129-1131.

Fiorentino F, Treasure T (2013b) Pulmonary metastasectomy for colorectal cancer: making the case for a randomized controlled trial in the zone of uncertainty. I Thorac Cardiovasc Surg 146: 748-752.

Fiorentino F, Vasilakis C, Treasure T (2011) Clinical reports of pulmonary metastasectomy for colorectal cancer: a citation network analysis. Br J Cancer 104: 1085-1097.

Glasziou P, Chalmers I, Rawlins M, McCulloch P (2007) When are randomised trials unnecessary? Picking signal from noise. BMJ 334 349-351.

Gonzalez M, Poncet A, Combescure C, Robert J, Ris HB, Gervaz P (2013) Risk factors for survival after lung metastasectomy in colorectal cancer patients: a systematic review and meta-analysis. Ann Surg Oncol 20: 572-579.

Greenberg SA (2009) How citation distortions create unfounded authority: analysis of a citation network. BMJ 339: b2680.

Pfannschmidt J, Dienemann H, Hoffmann H (2007) Surgical resection of pulmonary metastases from colorectal cancer: a systematic review of published series. Ann Thorac Surg 84: 324-338.

Pfannschmidt J, Hoffmann H, Dienemann H (2010) Reported outcome factors for pulmonary resection in metastatic colorectal cancer. J Thorac Oncol 5: S172-S178.

Primrose JN, Perera R, Gray A, Rose P, Fuller A, Corkhill A, George S, Mant D (2014) Effect of 3 to 5 years of scheduled CEA and CT follow-up to detect recurrence of colorectal cancer: the FACS randomized clinical trial. JAMA 311: 263-270.

Renaud S, Romain B, Falcoz PE, Olland A, Santelmo N, Brigand C, Rohr S, Guenot D, Massard G (2015) KRAS and BRAF mutations are prognostic biomarkers in patients undergoing lung metastasectomy of colorectal cancer. Br J Cancer 112: 720-728.

Simms L, Barraclough H, Govindan R (2013) Biostatistics primer: what a clinician ought to know-prognostic and predictive factors. J Thorac Oncol 8 808-813.

Treasure T, Mineo T, Ambrogi V, Fiorentino F (2015) Survival is higher after repeat lung metastasectomy than after a first metastasectomy: too good to be true? I Thorac Cardiovasc Surg 149(5): 1249-1252.

Treasure T, Monson K, Fiorentino F, Russell C (2014) The CEA Second-Look Trial: a randomised controlled trial of carcinoembryonic antigen prompted reoperation for recurrent colorectal cancer. BMJ Open 4: e004385.

Treasure T, Utley M (2007) Statistics for the rest of us: Ten traps for the unwary in surgical series: a case study in mesothelioma reports. J Thorac Cardiovasc Surg 133: 1414-1418.

Utley M, Treasure T (2008) The use of scoring systems in selecting patients for lung resection: work-up bias comes full-circle. Thorac Surg Clin 18: $107-112$.

Utley M, Treasure T, Linklater K, Moller H (2008) Better out than in? The resection of pulmonary metastases from colorectal tumours. In Operations Research for Health Care Engineering: Proceedings of the 33rd International Conference on Operational Research Applied to Health Services, Xie X, Lorca F, Marcon E (eds), Saint-EtiennePublications de l'Universitaire de Saint-Etienne, pp 493-500.

*Correspondence: T Treasure; E-mail: Tom.treasure@gmail.com 